SLAC-R-597

October 2006

\title{
A Search for the Rare Leptonic B- to tau- anti-neutrino Recoiling against B+ to Decays to anti-D*0 l+ Lepton-neutrino.
}

\author{
Mousumi Datta
}


A Search for the Rare Leptonic B DeCay $B^{-} \rightarrow \tau^{-} \bar{\nu}_{\tau}$ Recolling Against $B^{+} \rightarrow \bar{D}^{* 0} \ell^{+} \nu_{\ell}$

by

\begin{abstract}
Mousumi Datta
A dissertation submitted in partial fulfillment of the requirements for the degree of
\end{abstract}

DOCTOR OF PHILOSOPHY

(PHYSICS)

at the

UNIVERSITY OF WISCONSIN - MADISON

2005 


\begin{abstract}
This thesis describes a search for the decay $B^{-} \rightarrow T^{-} \bar{\nu}_{\tau}$ in $231.8 \times 10^{6} \Upsilon(4 \mathrm{~S})$ decays recorded with the BABAR detector at the SLAC PEP-II B-Factory. A sample of events with one reconstructed exclusive semi-leptonic $B$ decay $\left(B^{+} \rightarrow \vec{D}^{* 0} \ell^{+} \nu_{\ell}\right)$ is selected, and in the recoil a search for $B^{-} \rightarrow \tau^{-} \nu_{\tau}$ signal is performed in the following $\tau$ decay modes: $\tau^{-} \rightarrow e^{-} \bar{\nu}_{e} \nu_{\tau+} \tau^{-} \rightarrow \mu^{-} \bar{\nu}_{\mu} \nu_{\tau}, \tau^{-} \rightarrow \pi^{-} \nu_{\tau}, \tau^{-} \rightarrow \pi^{-} \pi^{0} \nu_{\tau}$, and $\tau^{-} \rightarrow \pi^{-} \pi^{+} \pi^{-} \nu_{\tau}$. We find no evidence of signal, and we set a preliminary upper limit on the branching fraction of $\mathcal{B}\left(B^{-} \rightarrow \tau^{-} \bar{\nu}_{7}\right)<2.8 \times 10^{-4}$ at the $90 \%$ confidence level (CL). This result is then combined with a statistically independent $B A B A R$ search for $B^{-} \rightarrow \tau^{-} \bar{\nu}_{r}$ to give a combined preliminary limit of $\mathcal{B}\left(B^{-} \rightarrow \tau^{-} \bar{\nu}_{+}\right)<2.6 \times 10^{-4}$ at $90 \% \mathrm{CL}$.
\end{abstract}




\section{Acknowledgements}

First, I would like to thank Professor Sau Lan Wu for her support, without which this thesis work would not have been possible. She has provided constant help and guidance throughout my graduate career. I would also like to thank my thesis advisor Professor Yibin Pan. Despite being seriously ill for a long period of time, he has given me advice and support whenever I needed.

I am also thankful to all the members of the BABAR collaboration, who have contributed to the successful completion of the analysis. I would like to especially thank the conveners of the Leptonic Bottom and Charm analysis working group (AWG) Steve Robertson, Rafe Schindler, Gregory Dubois-Felsmann, and Steve Sekula for their input. All the suggestions and inputs from the AWG members and the collaboration during the review processes have been invaluable. I am grateful to the committee which has tirelessly reviewed this work for BABAR: Dieter Best, Fabrizio Bianchi, Homer Neal, and Eli Rosenberg.

I would also like to thank all my colleagues of the Wisconsin BABAR groups. The significance of numerous conversation with them, including those which are not related to physics, cannot be underestimated. I am thankful for their friendship, and all the help they have provided me over the last four and a half years.

Finally I would like to thank my parents Sanjit Datta and Mira Datta, my sister Susmita Datta. Without their inspiratlon, love, and support, I would not be sitting here writing the last sentence of my thesis. 


\section{Contents}

1 Introduction $\quad 1$

1.1 The Standard Model . . . . . . . . . . . . . . . . . . . . 2

1.2 The Theory of Electroweak Interaction $\ldots \ldots \ldots \ldots$

1.2 .1 Electroweak Unification . . . . . . . . . . . . . . . . 3

1.2 .2 The Higgs Mechanism . . . . . . . . . . . . . 7

1.2 .3 Quark Mixing . . . . . . . . . . . . . . . . . . . . 9 9

1.2 .4 Electroweak Effective Lagrangian . . . . . . . . . . . . . 11

1.3 Limitations of the $\mathrm{SM} \ldots \ldots \ldots \ldots \ldots$

1.4 Experimental Motivation . . . . . . . . . . . . . . . . . 14

1.4.1 The Purely Leptonic Decay $B^{-} \rightarrow \tau^{-} \bar{\nu}_{r}$ in the SM $\ldots . . . .15$

1.4.2 Direct Measurement of the $B$ Meson Decay Constant . . . . . . . . 19

1.4 .3 Constraints on the CKM parameters .............. 20

1.4.4 Supersymmetry and $B^{-} \rightarrow \tau^{-} \bar{\nu}_{+}$Decay $\ldots \ldots \ldots . . . . .20$

1.5 Other Searches for $B^{-} \rightarrow \tau^{-} \bar{\nu}_{\tau}$ Decay . . . . . . . . . . . 21

2 The PEP-II $B$ Factory and The BABAR Detector 22

2.1 The PEP-II $B$ Factory . . . . . . . . . . . . . . . . . . . 23

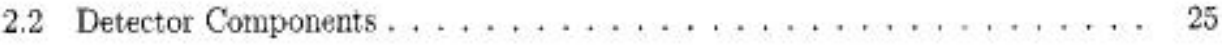

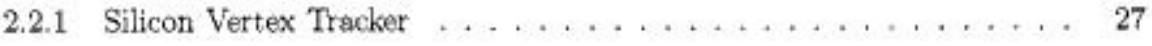

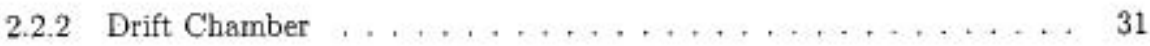

2.2 .3 Detector of Internally Reflected Cherenkov Light $\ldots \ldots \ldots . . . .35$

2.2.4 Electromagnetic Calorimeter . . . . . . . . . . . . . . 40

2.2 .5 Instrumented Flux Return . . . . . . . . . . . . . . 44

2.3 Electronics, Trigger, Data Acquisition, and Online Computing . . . . . 47 


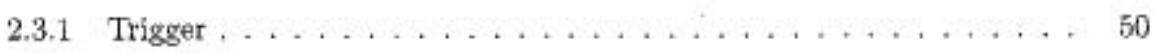

3 Analysis Strategy for $B^{-} \rightarrow \tau^{-} \bar{\nu}_{T}$ Search $\quad 52$

3.1 The $\operatorname{Tag} B \ldots \ldots \ldots \ldots \ldots$

3.2 The Signal-side . . . . . . . . . . . . . . . . 55

3.3 Analysis Overview ........................ 57

4 Data and Monte Carlo Samples $\quad 58$

5 Event Reconstruction $\quad 61$

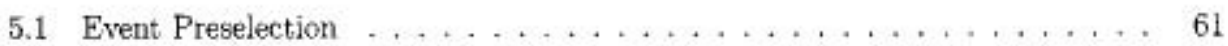

$5.2 \operatorname{Tag} B$ Reconstruction $\ldots \ldots \ldots \ldots \ldots \ldots \ldots \ldots \ldots \ldots \ldots . \ldots \ldots \ldots$

$5.2 .1 \quad \bar{D}^{0}$ Reconstruction .................... 62

$5.2 .2 \bar{D}^{* 0}$ Reconstruction ........................ 64

5.2 .3 Lepton Selection . . . . . . . . . . . . . . . . . 65

$5.2 .4 \bar{D}^{* 0} \ell^{+} \nu_{\ell}$ Reconstruction $\ldots \ldots \ldots \ldots . \ldots . \ldots . \ldots$

5.2.5 The Best $B_{\mathrm{sl}}^{+} \rightarrow \bar{D}^{* 0} \ell^{+} \nu_{\ell}$ Candidate Selection $\ldots \ldots \ldots \ldots$

5.2 .6 Additional Requirements for $B_{s 1}^{+}$Selection ... . . . . . . 71

$5.3 B_{\mathrm{si}}^{+}$Efficiency and Yield $\ldots \ldots \ldots \ldots \ldots \ldots \ldots$

$\begin{array}{llr}6 & \text { Signal Selection } & 83\end{array}$

6.1 Variables for Signal Selection . . . . . . . . . . . . . . . . 85

6.1 .1 Signal-side Track Multiplicity . . . . . . . . . . . 85

6.1 .2 Signal-side $\pi^{0}$ Multiplicity . . . . . . . . . . . . . 85

6.1.3 Particle Identification for the Signal-side Track(s) . . . . . . . 86

6.1.4 Momentum of the Signal Track(s) in the Center-of-mass Frame . . . 87

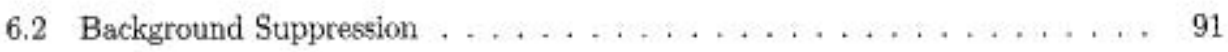


6.2.1 Removal of Un-modeled Excess Events . . . . . . . . . . . . 91

6.2 .2 Continuum Background Suppression $\ldots \ldots \ldots \ldots \ldots \ldots . . .93$

6.2.3 Requirements on Intermediate Resonances . . . . . . . . . . 94

6.3 The Signal Defining Quantity $E_{\text {extrs }} \ldots \ldots \ldots \ldots \ldots . \ldots \ldots$

6.4 Summary of Signal Selection Criteria by Selection Channel . . . . . . 105

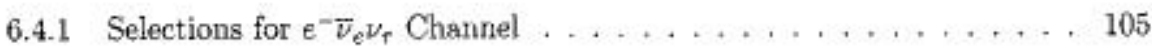

6.4.2 Selection for $\mu^{-} \bar{\nu}_{\mu} \nu_{\tau}$ Channel ............. 105

6.4 .3 Selection for $\pi^{-} \nu_{\tau}$ Channel . . . . . . . . . . . 106

6.4.4 Selection for Misidentified-Lepton Channel . . . . . . . . . . 107

6.4.5 Selection for $\pi^{-} \pi^{0} v_{\tau}$ Channel $\ldots \ldots \ldots \ldots \ldots \ldots . \ldots \ldots$

6.4.6 Selection for $\pi^{-} \pi^{+} \pi^{-} \nu_{\tau}$ Channel . . . . . . . . . . . . . . 109

$\begin{array}{lll}7 & \text { Signal Efficiency and Background Estimate } & 110\end{array}$

7.1 Signal Efficiency . . . . . . . . . . . . . . . . 110

7.2 Background Expectation from MC $\ldots \ldots \ldots \ldots \ldots \ldots \ldots \ldots$

7.3 Background Estimation from Data Side Band . . . . . . . . . . . 132

8 Validation and Systematics 136

8.1 Control Sarnples. . . . . . . . . . . . . . . . . 137

8.1 .1 Double-tag Sample . . . . . . . . . . . . . 138

8.1.2 Two Signal-side Tracks Sample . . . . . . . . . . . 140

8.1 .3 Non-zero Net Charge Sample . . . . . . . . . . . 140

8.1 .4 Three Signal-side Tracks with Wrong PID . . . . . . . . . 140

$8.1 .5 \Delta M$ Side-band Sample . . . . . . . . . . . . . . . . . 141

8.2 Validation of the $E_{\text {axtra }}$ Simulation $\ldots \ldots \ldots \ldots \ldots 14$

8.2.1 Validation of the $E_{\text {extra }}$ Simulation in the Signal MC . . . . . 141 
8.2.2 Validation of the Background Estimation from $E_{\text {extra }}$ Side-band Ex-

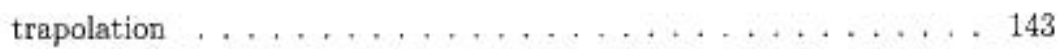

8.3 Correction and Systematic Uncertainty on the $B_{\mathrm{s} 1}^{+}$Reconstruction Efficiency 148

$8.3 .1 \quad B_{\mathrm{sl}}^{+}$Efficiency Correction . . . . . . . . . . . . . . . 148

8.3.2 Systematic Uncertainty on the $B_{\mathrm{gl}}^{+}$Reconstruction Efficiency . . . . 150

8.4 Corrections and Systematic Uncertainties on Signal-side Selection Efficiencies 151

8.4.1 Correction and Systematic Uncertainty from $E_{\text {extra }}$ Simulation . . . 152

8.4.2 Correction and Systematic Uncertainty from $\pi^{\circ}$ Reconstruction Eff-

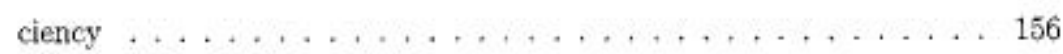

8.4.3 Correction and Systematics from Tracking Efficiency . . . . . 157

$8,4.4$ Correction and Systematic Uncertainty from PID . . . . . . . 157

8.4.5 Systematic Uncertainty from Fisher Discriminant . . . . . . . 162

8.5 Corrections and Systematic Uncertainties on Background Estimations . . . 163

8.6 Systematic Uncertainty on Number of $B^{+} B^{-}$Events $\ldots \ldots \ldots . . . \ldots 164$

8.7 Summary of Corrections and Systematic Uncertainties . . . . . . 167

8.8 Incorpotating Systematic Uncertainties . . . . . . . . . . . . 168

9 Statistical Procedure for Branching Fraction Extraction $\quad 171$

9.1 Upper Limit Expectation . . . . . . . . . . . . . . . . . . . . . 173

10 Physics Results from Data $\quad 176$

10.1 Branching Fraction Upper Limit . . . . . . . . . . . . . . . 177

10.2 Combining Results with BABAR Analysis for $B^{-} \rightarrow \tau^{-} \bar{\nu}_{\tau}$ Search Using Hadronic

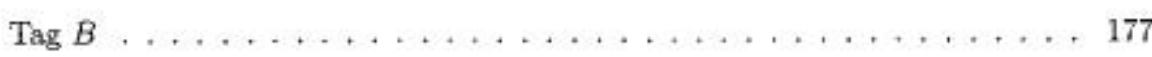

10.3 Constraints from Combined Branching Fraction Upper Limit . . . . . . . 179

10.3 .1 Constraint on $f_{B} \ldots \ldots \ldots \ldots \ldots \ldots$ 
10.4 Constraints on CKM Parameters $\ldots \ldots \ldots \ldots \ldots$

10.4.1 Constraints on Charged Higgs Parameters . . . . . . . . . . 181

11 Summary $\quad \cdot \quad 182$

A Track, Photon, and $\pi^{0}$ List Definitions 183

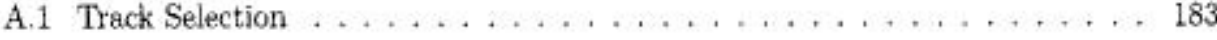

A.2 Photon Selection . . . . . . . . . . . . . . . . . 184

A.3 Clean Photon Selection $\ldots \ldots \ldots \ldots \ldots \ldots \ldots$

A.4 Neutral Cluster Selection from Reference $[65] \ldots \ldots \ldots \ldots$. . . . . . 185

A.5 $\pi^{0}$ selection $\ldots \ldots \ldots \ldots \ldots \ldots \ldots \ldots \ldots$

B Difference in Neutral Systematics Between SP5 and SP6 Signal MC for $\pi^{-} \pi^{+} \pi^{-} \nu_{\tau}$ Selection 


\section{List of Figures}

1 In the SM purely leptonic decay $B^{-} \rightarrow \ell^{-} \bar{\nu}_{\ell}$ proceeds via quark annihilation into a $W^{-}$boson. . . . . . . . . . . . . . . . 15

2 The right-handed helicity of the outgoing leptons in the $B^{-} \rightarrow \ell^{-} \bar{\nu}_{\ell}$ decay. . 18

3 The layout of the interaction region of the PEP-II $B$ Factory. . . . . . 25

4 Longitudinal section of the BABAR detector. . . . . . . . . . . 26

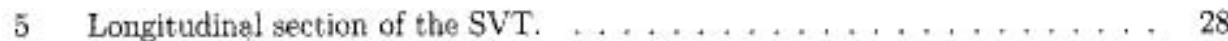

6 Transverse section of the SVT . . . . . . . . . . . . . . . . . 29

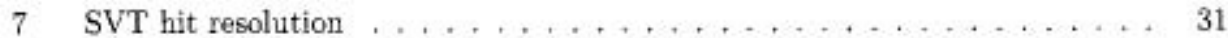

8 Longitudinal section of the DCH $\ldots \ldots \ldots \ldots \ldots \ldots \ldots$

9 Schematic layout of drift cells for the four innermost supet-layers . . . . . 33

10 The drift time versus distance relation, and position resolution of the DCH. . 35

11 Measurement of $d E / d x$ in the DCH $\ldots \ldots \ldots \ldots \ldots \ldots \ldots$

12 Schematics of the DIRC fused silica radiator bar and imaging region . . . . 37

13 Schematic of the DIRC mechanical support structure. . . . . . . . . . 38

14 The difference between the measured and expected Cherenkov angle . . . . . 39

15 Expected $\pi-\mathrm{K}$ separation in $B^{0} \rightarrow \pi^{+} \pi^{-}$events versus track momentum . . . 40

16 A longitudinal cross section of the EMC $\ldots \ldots \ldots \ldots, \ldots \ldots, \ldots$

17 A schematic of the wrapped CsI(T1) crystal. . . . . . . . . . . 42

18 The energy resolution of the EMC $\ldots \ldots \ldots \ldots \ldots \ldots \ldots$

19 The angular resolution of the EMC $\ldots \ldots \ldots \ldots \ldots \ldots \ldots$

20 Overview of the IFR $\ldots \ldots \ldots \ldots \ldots \ldots \ldots \ldots \ldots \ldots \ldots$

21 Cross section of a planar RPC $\ldots \ldots \ldots \ldots \ldots \ldots \ldots \ldots \ldots$

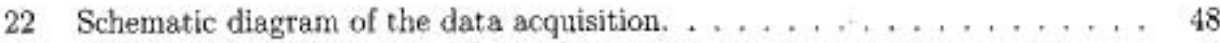


23 Schematic diagram of the Front-End Electronics . . . . . . . . . 49

24 A cartoon diagram of $B^{-} \rightarrow \tau^{-} \bar{\nu}_{\tau}$ in the $B$ Factory environment. $\ldots \ldots, 53$

25 Major Feynman diagrams for $\bar{b} \rightarrow \bar{c}$ transition. . . . . . . . . . . . 54

26 Distributions of quantities that are used for $B_{\mathrm{sl}}^{+}$selection, for correctly reconstructed $\bar{D}^{* 0} \ell^{+} \nu_{\ell}$ candidates in signal MC . . . . . . . . . . 66

27 Number of reconstructed $\bar{D}^{* 0} \ell^{+}$candidates per event before the best candidate

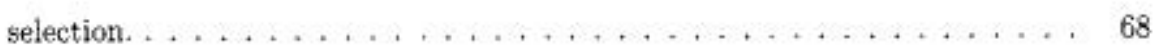

28 The normalized histograms of $\Delta M$ and $\bar{D}^{0}$ mass distributions for correctly reconstructed $\bar{D}^{* 0} \ell^{+} \nu_{t}$ candidates. . . . . . . . . . . . 69

29 Two dimensional scatter plot of $\Delta M$ versus difference between reconstructed and nominal $\bar{D}^{0}$ mass for correctly reconstructed $\bar{D}^{* 0} \ell^{\dagger} \nu_{\ell}$ candidates. . . . 70

$30 \quad \bar{D}^{0}$ mass distributions for the $B_{s}^{+}$candidate. $\ldots \ldots \ldots \ldots 73$

31 Comparison of the $\Delta M$ distributions between data and MC. . . . . . . 74

32 The CM-frame momentum for the lepton from $\bar{D}^{* 0} \ell^{+} \nu_{\ell} \ldots \ldots \ldots \ldots$

33 The angle between $\bar{D}^{0}$ and neutral from $\bar{D}^{* 0} \ell^{+} \nu_{\ell}$ in CM-frame between data and $\mathrm{MC} \ldots \ldots \ldots \ldots \ldots \ldots \ldots$

34 Net event charge distributions. . . . . . . . . . . . . . . 77

35 Signal-side Charged Tracks and Good TracksLoose multiplicities. . . . . . . 89

36 Signal-side $\pi^{0}$ multiplicities. . . . . . . . . . . . . . . . 90 90

$37 R_{2 a l l}$ and Charged Tracks multiplicity of events with electron signal track . . 96

38 The missing mass and CM-frame momentum of the signal-side track for the $e^{-\bar{\nu}_{6} \nu_{\tau} \text { channel } \ldots \ldots \ldots \ldots \ldots \ldots \ldots \ldots \ldots \ldots \ldots}$

39 The CM-frame momentum of the signal-side track and missing mass distributions for the $e^{-} \bar{\nu}_{e} \nu_{\tau}$ channel after removal of un-modeled processes. . . . . . 98

40 The input variables for the Fisher discriminant. . . . . . . . . . . . . 99 
41 The output of the Fisher discriminant. . . . . . . . . . 100

42 Invariant mass and CM-frame momentum of the $\pi^{-}$and $\pi^{0}$ combination, and $\cos \theta_{r-\rho}$ distributions for $\pi^{-} \pi^{0} \nu_{\tau}$ channel. . . . . . . . . . . . 101

43 Invariant mass and CM-frame momentum of the three signal tracks, and $\cos \theta_{\tau, \text { hnd }}$ for $\pi^{-} \pi^{+} \pi^{-} \nu_{\tau}$ channel. . . . . . . . . . . . . 102

$44 E_{\text {extra }}$ distributions for $\bar{D}^{* 0}\left\{\bar{D}^{0} \gamma\right) e^{+} \nu_{e}$ events after the best candidate selection 104

45 The CM-frame momentum distributions for events in the $\mu^{-} \bar{\nu}_{\mu} \nu_{\tau}$ channel. . 106

46 The CM-frame momentum of the signal track in the $\pi^{-} \nu_{\tau}$ and misidentified-

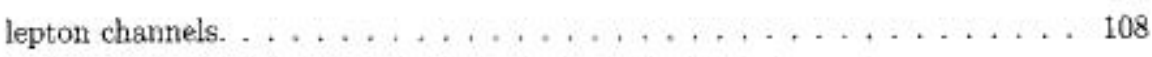

47 The $E_{\text {extra }}$ distributions for different selection channels in SP5 background and signal MC and Run 1-3 on-resonance data. . . . . . . . . . . 134

48 The $E_{\text {extra }}$ distributions for different selection channels in SP6 background and signal MC and Run 4 on-resonance data . . . . . . . . . . 135

49 The $E_{\text {extro }}$ distributions for double-tag events. . . . . . . . . . . 142

$50 E_{\text {extra }}(\mathrm{GeV})$ distributions for two signal-side tracks and $Q_{\text {net }} \neq 0$ control samples. ................................. 144

$51 E_{\text {extra }}(\mathrm{GeV})$ distributions for three signal-side tracks with wrong PID, and $\Delta M$ side-band control samples. . . . . . . . . . . . 145

52 The distribution of the number of photons with cluster energy above $30 \mathrm{MeV}, 152$

53 The efficiencies for various cut values on the Fisher output in double-tag events. 163

54 The nominal expected CL curve and $-2 \log (Q)$ distributions as a function of

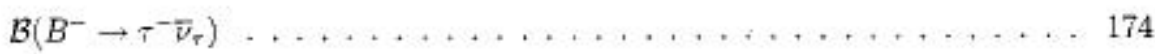

55 The CL curve and $-2 \log Q$ as a function of $\mathcal{B}\left(B^{-} \rightarrow T^{-} \bar{\nu}_{r}\right)$ for observed events in data $\ldots \ldots \ldots \ldots \ldots \ldots \ldots \ldots \ldots \ldots$ 
$56 \mathrm{CL}$ and $-2 \log \mathrm{Q}$ distributions as functions of $\mathcal{B}\left(B^{-} \rightarrow \tau^{-} \bar{\nu}_{\tau}\right)$ for combined $B_{\mathrm{s}}^{+}$ and hadronic $\operatorname{tag} B$ samples. . . . . . . . . . . . . . . 180

57 Constraints on $(\bar{\rho}, \pi)$ parameter plane, obtained by the CKMfitter Group, from $\mathcal{B}\left(B^{-} \rightarrow \tau^{-} \nu_{\tau}\right)$ and $\Delta m_{d^{*}} \ldots \ldots \ldots \ldots \ldots 18 \ldots \ldots \ldots$ 


\section{List of Tables}

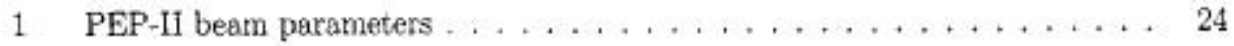

2 Production cross-sections at $\sqrt{s}=M(\Upsilon(4 \mathrm{~S})) \ldots \ldots \ldots \ldots \ldots . \ldots 24$

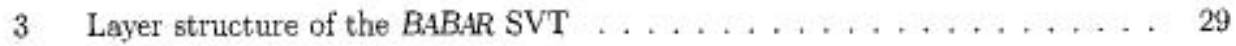

4 Properties of helium-isobutane gas mixture $\ldots \ldots \ldots \ldots \ldots \ldots, 34$

5 Branching fractions for the major $\tau$ decay modes. . . . . . . . . . 52

6 Branching fraction of the decays involved in $B^{+} \rightarrow \bar{D}^{* 0} \ell^{+} \nu_{\ell}$ reconstruction . 56

7 Data samples used in the anslysis. . . . . . . . . . . . . . . . 59

8 MC samples used in the analysis. . . . . . . . . . . . . 60

$9 B_{\mathrm{k1}}^{+}$selection efficiencies for " $B^{-} \rightarrow \tau^{-} \bar{\nu}_{\tau}$ versus generic" SP5 and SP6 MC. , 80

10 Yields in different $B_{\mathrm{al}}^{+}$reconstruction modes for " $B^{-} \rightarrow \tau^{-} \bar{\nu}_{\tau}$ versus generic" MC, after applying all $B_{\mathrm{sl}}^{+}$selections, except, $Q_{\text {net }}$ requirement. ' . . . . 81

11 Comparisons of number of events, and marginal and cumulative efficiencies of on-resonance data and generic MC normalized to data luminosity, . . . . . 81

12 Comparisons of number of events, and marginal and cumulative efficiencies of off-resonance data and generic MC normalized to data luminosity. . . . . 82

13 The $E_{\text {extrat }}$ requirements for the six selection channels . . . . . . . . . 104

14 The summary of selection criteria for the six signal channels. . . . . . . . 111

15 Signal-side selection efficiencies for $\tau^{-} \rightarrow e^{-} \bar{\nu}_{e} \nu_{\tau}$ and $\tau^{-} \rightarrow \mu^{-} \bar{\nu}_{\mu} \nu_{\tau}$ channels for SP5 signal MC $\ldots \ldots \ldots \ldots \ldots \ldots \ldots \ldots \ldots \ldots \ldots \ldots$

16 Signal-side selection efficiencies for $\tau^{-} \rightarrow \pi^{-} \nu_{+}$and misidentified-lepton channels for SP5 signal MC . . . . . . . . . . . . . . . . . . . 114

17 Signal-side selection efficiencies for $\tau^{-} \rightarrow \pi^{-} \pi^{0} \nu_{\tau}$ channel for SP5 signal MC. 115 
18 Signal-side selection efficiencies for $\tau^{-} \rightarrow \pi^{-} \pi^{+} \pi^{-} \nu_{+}$channel for SP5 signal

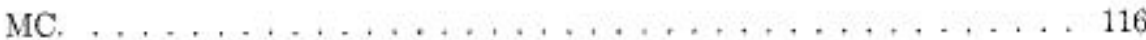

19 Signal-side selection efficiencies for $\tau^{-} \rightarrow e^{-} \nu_{c} \nu_{\tau}$ and $\tau^{-} \rightarrow \mu^{-} \bar{\nu}_{\mu} \nu_{+}$channels for SP6 signal MC. . . . . . . . . . . . . . . . . . 117

20 Signal-side selection efficiencies for $\tau^{-} \rightarrow \pi^{-} \nu_{r}$ and misidentified-lepton channels for $\mathrm{SP6}$ signal MC . . . . . . . . . . . . . . . 118

21 Signal-side selection efficiencies for $\tau^{-} \rightarrow \pi^{-} \pi^{0} \nu_{\tau}$ channel for SP6 signal MC. 119

22 Signal-side selection efficiencies for $\tau^{-} \rightarrow \pi^{-} \pi^{+} \pi^{-} \nu_{\tau}$ channel for SP6 signal

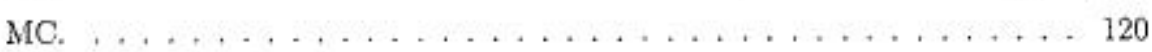

23 Signal-side selection efficiencies of $B^{-} \rightarrow \tau^{-} \bar{\nu}_{\tau}$ events from signal MC. . . 121

24 Yields and efficiencies of the signal-side selections for $e^{-} \bar{\nu}_{e} \nu_{\tau}, \mu^{-} \bar{\nu}_{\mu} \nu_{\tau}$, and $\pi^{-} \nu_{\tau}$ channels in Run 1-3 on-peak data and SP5 background MC. . . . . . 123

25 Yields and efficiencies of the signal-side selections for misidentified-lepton and $\pi^{-} \pi^{0} \nu_{\tau}$ channels in Run 1-3 on-peak data and SP5 background MC. . . . . 124

26 Yields and efficiencies of the signal-side selections for $\pi^{-} \pi^{+} \pi^{-} \nu_{\tau}$ channel in Run 1-3 on-peak data and SP5 background MC . . . . . . . . . . . . 125

27 Yields and efficiencies of the signal-side selections for $e^{-} \bar{\nu}_{e} \nu_{\tau+} \mu^{-} \bar{\nu}_{\mu} \nu_{+}$, and $\pi^{-} \nu_{\tau}$ channels in Run 4 on-peak data and SP6 background MC . . . . . 126

28 Yields and efficiencies of the signal-side selections for misidentified-lepton and $\pi^{-} \pi^{0} \nu_{\tau}$ channels in Run 4 on-peak data and SP6 background MC . . . . 127

29 Yields and efficiencies of the signal-side selections for $\pi^{-} \pi^{+} \pi^{-} \nu_{+}$channel in Run 4 on-peak data and SP6 background MC . . . . . . . . . . 128

30 Expected final background and signal yields estimated from SP5 background and signal $\mathrm{MC}$ events scaled to $110.8 \mathrm{fb}^{-1} \ldots \ldots \ldots \ldots \ldots$ 
31 Expected final background and signal yields estimated from SP6 background and signal MC events scaled to $99.8 \mathrm{fb}^{-1} \ldots \ldots \ldots \ldots \ldots \ldots$

32 Expected final background and signal yields estimated from all background and signal $\mathrm{MC}$ scaled to $210.6 \mathrm{fb}^{-1} \ldots \ldots \ldots \ldots \ldots \ldots \ldots \ldots$

33 Background estimation for the different selection channels from $E_{\text {extra }}$ sideband extrapolation $\ldots \ldots \ldots \ldots \ldots \ldots \ldots \ldots \ldots \ldots \ldots \ldots$

34 Yields of double-tag events in data and MC . . . . . . . . . . 139

35 Validation of background estimation in the signal region from various control samples in Run $1-3$ data . . . . . . . . . . . . . . 146

36 Validation of background estimation in the signal region from various control samples in Run 4 data . . . . . . . . . . . . . 147

37 Correction factors for $B_{a t}^{+}$efficiency and yield. . . . . . . . . . 149

38 Comparison of the average multiplicity for clusters with different energy scale between $\mathrm{MC}$ and data $\ldots \ldots \ldots \ldots \ldots \ldots \ldots \ldots \ldots \ldots$

39 Correction factors and systematic uncertainties for signal efficiencies due to

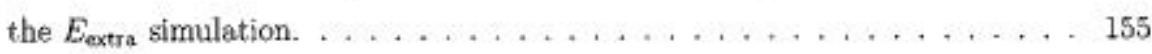

40 Correction factors and systematics due to $\pi^{0}$ killing in signal MC. . . . . 157

41 Estimated final signal yields before and after PID correction for channels with

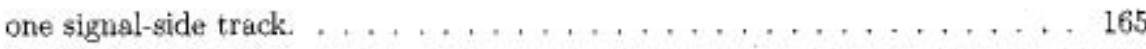

42 Estimated final signal yield for $\pi^{-} \pi^{+} \pi^{-} \nu_{+}$channel before and after PID correction. . . . . . . . . . . . . . . . . . . . 166

43 Correction factors and systematics for background estimation due to $E_{\text {extra }}$ simulation. . . . . . . . . . . . . . . . . 166

44 Corrections to signal-side selection efficiencies from varfous sources. . . . . 167 
45 The systematic uncertainties from various sources that are associated with signal-side selections. . . . . . . . . . . . . . . 168

46 Summary of the efficiency related corrections and systematics, . . . . . . 169

47 The background estimation with corrections and systematic uncertainties incorporated. ................................ 170

48 Nominal expected upper limits $(90 \% \mathrm{CL})$ for individual and combinations of selection channel(s). ............................ 175

49 Observed number of on-resonance data events and expected background events in the signal region. . . . . . . . . . . . . . 176

50 Upper limits for individual selection channels. . . . . . . . . . . 179

51 The MC information of the neutral clusters contributing to $E_{\text {extra }}$ for signal MC events satisfying the selection cuts for $\pi^{-} \pi^{+} \pi^{-} \nu_{\tau}$ channel. . . . . . . . 189 